\title{
APPROXIMATE IDENTITIES AND PARACOMPACTNESS
}

\author{
R. A. FONTENOT AND R. F. WHEELER \\ Dedicated to Heron $S$. Collins, our teacher and friend
}

\begin{abstract}
Let $X$ denote a locally compact Hausdorff space and $C_{b}(X)$ the algebra of continuous complex-valued functions on $X$. The main result of this paper is that $X$ is paracompact if and only if $C_{0}(X)$, the subalgebra of $C_{b}(X)$ consisting of functions which vanish at infinity, has an approximate identity which is a relatively compact subset of $C_{b}(X)$ for the weak topology of the pairing of $C_{b}(X)$ with its strict topology dual.
\end{abstract}

Throughout this paper $X$ denotes a locally compact Hausdorff space, $C_{b}(X)$ denotes the algebra of continuous complex-valued functions on $X$, equipped with the strict topology $\beta$ introduced by Buck [1] and studied by numerous others (for example, see $[5,11]$, and the surveys $[2,14])$, and $C_{0}(X)$ denotes the algebra of continuous complex-valued functions which vanish at infinity on $X$. Buck proved that the $\beta$-dual of $C_{b}(X)$ is $M_{t}(X)$, the space of bounded tight (inner regular by compact sets) Borel measures on $X$. By the weak topology on $C_{b}(X)$, we mean the topology $\sigma\left(C_{b}(X), M_{t}(X)\right)$.

An approximate identity for $C_{0}(X)$ is a uniformly bounded net $\left(f_{\alpha}\right)_{\alpha \in A}$ in $C_{0}(X)$ such that $\left\|h f_{\alpha}-h\right\| \rightarrow 0$ for each $h \in C_{0}(X)$. An approximate identity is said to be well-behaved (a WBAI) if the following conditions hold: (1) $0 \leq f_{\alpha} \leq 1$ for all $\alpha \in A$; (2) $\alpha_{1}<\alpha_{2} \Rightarrow f_{\alpha_{1}} f_{\alpha_{2}}=f_{\alpha_{1}}$; and (3) if $\alpha \in A$ and $\left(\alpha_{n}\right)$ is a strictly increasing sequence in $A$, then there exists a positive integer $N$ such that $f_{\alpha} f_{\alpha_{n}}=f_{\alpha} f_{\alpha_{N}}$ for $n \geq N$. Taylor [12] introduced the notion of WBAI and showed that if $X$ is paracompact, then $C_{0}(X)$ has a WBAI. We shall use two other abbreviations: WCAI, to stand for an approximate identity for $C_{0}(X)$ that is relatively weakly compact in $C_{b}(X)$, and TBAI, to denote a $\beta$-totally bounded (= equicontinuous) approximate identity for $C_{0}(X)$.

Collins and Fontenot [4] studied various classes of approximate identities for $C_{0}(X)$, relationships between these classes, and implications for the topological properties of $X$. Building on earlier work of Collins and Dorroh [3], they showed that $C_{0}(X)$ has a TBAI if and only if $X$ is paracompact. Subsequently, Wheeler $[\mathbf{1 3}]$ showed that paracompactness is also equivalent to existence of a WBAI for $C_{0}(X)$. His argument uses Stone-Cech compactifications and a set-theoretic lemma due to A. Hajnal. Collins and Fontenot also considered WCAI's: they gave an example, the space of countable ordinals, of a space $X$ such that $C_{0}(X)$ has no WCAI and left open the problem of characterizing spaces $X$ such that $C_{0}(X)$ has a WCAI.

In this paper we do three things. First, and principally, we show that $C_{0}(X)$ has a WCAI if and only if $X$ is paracompact, and we observe that our argument also

Received by the editors November 25, 1985 .

1980 Mathematics Subject Classification (1985 Revision). Primary 46E25; Secondary 46J10, 54D18. Key words and phrases. Approximate identity, paracompact, weakly compact. 
applies in the WBAI case to give a new and simpler proof of Wheeler's result [13, Theorem 2.1]. Second, by a direct argument that does not appeal to paracompactness of $X$, we prove that if $C_{0}(X)$ has a WCAI, then it has a WBAI and note that this result gives a second proof of our main theorem. Since a TBAI is a WCAI, we also get a new proof that $C_{0}(X)$ has a TBAI if and only if $X$ is paracompact. Third, we give an example to show that a WBAI or WCAI need not contain a subnet which is a TBAI. Thus we show that a plausible approach to both the WBAI and WCAI characterization problems will not work.

We begin by mentioning two (essentially) known results about relatively weakly compact sets in $C_{b}(X)$. The first is this: A uniformly bounded subset $F$ of $C_{b}(X)$ is relatively weakly compact if and only if it is relatively pointwise compact if and only if it is quasi-equicontinuous [7, p. 269]. The second result is the content of the following lemma.

LEMMA 1. A uniformly bounded subset $F$ of $C_{0}(X)$ is relatively weakly compact in $C_{b}(X)$ if and only if every sequence in $F$ has a subsequence converging pointwise to a function in $C_{b}(X)$.

ProOF. Suppose that $F$ satisfies the condition on sequences. The Lebesgue Dominated Convergence Theorem then implies that every infinite subset of $F$ has a weak cluster point in $C_{b}(X)$. Since $\beta$ is complete, $F$ is relatively weakly compact [8, Proposition 2, p. 177].

Suppose that $F$ is relatively weakly compact. Then $F$ is certainly relatively countably compact in the topology of pointwise convergence on $C_{b}(X)$. Let $\left(f_{n}\right)$ be a sequence in $F$, and let $S=\bigcup_{n}\left\{x \in X: f_{n}(x) \neq 0\right\}$. Note that $S$ is $\sigma$ compact. Let $F \in C_{b}(X)$ be a pointwise cluster point of the sequence $\left(f_{n}\right)$. By [9, Theorem 2.1], there is a subsequence $\left(f_{n_{k}}\right)$ such that $f_{n_{k}}(x) \rightarrow f(x)$ for $x \in S$. But $f_{n_{k}}(x)=f(x)=0$ for $x \in X-S$. Thus $f_{n_{k}} \rightarrow f$ pointwise on $X$. This concludes the proof.

The proof of Theorem 3, our main result, uses a set-theoretical lemma due to Hajnal (see [13, Lemma 2.6]). We give here a much simpler proof due to Ruzsa [10].

LEMMA 2. Let $A$ be an infinite set, directed by $<$. Let $\left(K_{\alpha}\right)_{\alpha \in A}$ be a cover of a set $X$ with the following properties: (a) if $\alpha_{1}<\alpha_{2}$, then $K_{\alpha_{1}} \subset K_{\alpha_{2}}$; (b) if $\left(\alpha_{n}\right)$ is a strictly increasing sequence in $A$ and $\alpha \in A$, then there is a positive integer $N$ such that $K_{\alpha} \cap K_{\alpha_{n}}=K_{\alpha} \cap K_{\alpha_{N}}$ for $n \geq N$. Then there is a set $B$ and a family $\left(L_{\beta}\right)_{\beta \in B}$ of pairwise disjoint subsets of $X$ which covers $X$ and has the following properties: (1) for each $\beta \in B, \exists \alpha \in A$, such that $L_{\beta} \subset K_{\alpha}$; (2) for each $\alpha \in A$, there is a finite subset $F$ of $B$ such that $K_{\alpha} \subset \subset \bigcup_{\beta \in F} L_{\beta}$.

ProOF. Let $\ll$ be an arbitrary well-ordering of $A$. Let $B$ be the set of finite, nonempty subsets of $A$. Define a well-ordering, also denoted $\ll$, of $B$ as follows. Let $\beta=\left\{\alpha_{1}, \ldots, \alpha_{m}\right\}$ and $\beta^{\prime}=\left\{\alpha_{1}^{\prime}, \ldots, \alpha_{n}^{\prime}\right\}$, with $\alpha_{m} \ll \alpha_{m-1} \ll \cdots \ll \alpha_{1}$ and $\alpha_{n}^{\prime} \ll \alpha_{n-1}^{\prime} \ll \cdots \ll \alpha_{1}^{\prime}$, be two different elements of $B$. If $\alpha_{i}=\alpha_{i}^{\prime}$ for every $i \leq \min (m, n)$, let $\beta \ll \beta^{\prime}$ if and only if $m<n$. If not, let $j$ be the first index for which $\alpha_{j} \neq \alpha_{j}^{\prime}$, and let $\beta \ll \beta^{\prime}$ if and only if $\alpha_{j} \ll \alpha_{j}^{\prime}$. Note that if $\beta \subset \beta^{\prime}$, then $\beta \ll \beta^{\prime}$. 
Define, by induction on the cardinality of $\beta \in B$, an auxiliary function $g: B \rightarrow A$ in such a way that $g(\{\alpha\})=\alpha$ and if $\beta \subset \beta^{\prime}$, then $g(\beta)<g\left(\beta^{\prime}\right)$. Now let

$$
L_{\beta}=K_{g(\beta)}-\bigcup_{\beta^{\prime} \ll \beta} K_{g\left(\beta^{\prime}\right)} .
$$

The family $\left(L_{\beta}\right)$ consists of disjoint sets, has the same union as $\left(K_{\alpha}\right)$, and has the property that $L_{\beta} \subset K_{g(\beta)}$. All we need to prove is (2).

We prove by contradiction that each $K_{g(\beta)}$ is contained in a finite union of sets in the family $\left(L_{\beta}\right)$; this is sufficient for (2) since $K_{\alpha}=K_{g(\{\alpha\})}$. Suppose not, and let $\beta=\left\{\alpha_{1}, \ldots, \alpha_{n}\right\}$, with $\alpha_{n} \ll \cdots \ll \alpha_{1}$, be the first element of $B$ for which our proposition does not hold. Since $K_{g(\beta)}-L_{\beta} \subset \bigcup_{\beta^{\prime} \ll \beta} L_{\beta^{\prime}}$, there must be an infinite sequence $\beta_{1} \ll \beta_{2} \ll \cdots \ll \beta$ such that $K_{g(\beta)} \cap L_{\beta_{i}} \neq \varnothing$ for all $i$. Divide these $\beta_{i}$ 's into $n$ classes, the first consisting of those with all elements $\ll \alpha_{1}$ and the $j$-th, for $2 \leq j \leq n$, consisting of those $\beta_{i}$ 's which contaian $\alpha_{1}, \ldots, \alpha_{j-1}$ but not $\alpha_{j}$. One of these classes, say the $k$ th, must be infinite. Denote the $\beta_{i}$ 's of this class by $\delta_{1} \ll \delta_{2} \ll \cdots$.

Now let $\gamma_{i}=\delta_{1} \cup \cdots \cup \delta_{i}$. Then $\gamma_{i} \ll \beta$ since $\gamma_{i}$ contains $\alpha_{1}, \ldots, \alpha_{k-1}$ and all its other elements are $\ll \alpha_{k}$. Since $\gamma_{1} \subset \gamma_{2} \subset \cdots, g\left(\gamma_{1}\right)<g\left(\gamma_{2}\right)<\cdots$. Since $\left(K_{\alpha}\right)_{\alpha \in A}$ satisfies condition (b), there exists a positive integer $N$ such that for $n \geq N$

Thus, for $n \geq N$

$$
K_{g(\beta)} \cap K_{g\left(\gamma_{n}\right)}=K_{g(\beta)} \cap K_{g\left(\gamma_{N}\right)}
$$

$$
\begin{aligned}
\left(L_{\delta_{n}} \cap K_{g\left(\gamma_{N}\right)}\right) & \supset\left(L_{\delta_{n}} \cap K_{g\left(\gamma_{N}\right)} \cap K_{g(\beta)}\right)=\left(L_{\delta_{n}} \cap K_{g\left(\gamma_{n}\right)} \cap K_{g(\beta)}\right) \\
& \supset\left(L_{\delta_{n}} \cap K_{g\left(\delta_{n}\right)} \cap K_{g(\beta)}\right)=L_{\delta_{n}} \cap K_{g(\beta)} \neq \varnothing .
\end{aligned}
$$

Since the sets in the family $\left(L_{\beta^{\prime}}\right)_{\beta^{\prime} \in B}$ are pairwise disjoint, this implies that $K_{g\left(\gamma_{N}\right)}$ is not contained in a finite union of sets in the family. This is a contradiction since $\gamma_{N} \ll \beta$.

THEOREM 3. Let $X$ be a locally compact Hausdorff space. Then $C_{0}(X)$ has a $W C A I$ if and only if $X$ is paracompact.

PROOF. If $X$ is paracompact, the construction in [3, Theorem 4.2] yields a WCAI, since $\beta$-totally bounded sets are relatively $\beta$-compact.

Now suppose that $\left(f_{\alpha}\right)_{\alpha \in A}$ is a WCAI for $C_{0}(X)$. For each $\alpha \in A$, let $K_{\alpha}=$ $\left\{x: f_{\alpha}(x) \geq \frac{1}{2}\right\}$. The family $\left(K_{\alpha}\right)$ is a cover of $X$ consisting of compact sets. Introduce a (new) partial order on $A$ as follows: $\alpha_{1}<\alpha_{2}$ if and only if $f_{\alpha_{2}}>\frac{3}{4}$ on $K_{\alpha_{1}}$. It is evident that $A$ is a directed set and that if $\alpha_{1}<\alpha_{2}$, then $K_{\alpha_{1}} \subset$ $K_{\alpha_{2}}^{0} \subset K_{\alpha_{2}}$. Suppose that $\left(\alpha_{n}\right)$ is a strictly increasing sequence in $A$ with respect to the partial order just defined. Let $\Sigma=\bigcup_{n} K_{\alpha_{n}}$, and let $f$ be a weak and, hence, pointwise cluster point of $\left(f_{\alpha_{n}}\right)$ in $C_{b}(X)$. Since $f \geq \frac{3}{4}$ on $\Sigma$ and $f \leq \frac{1}{2}$ on $X-\Sigma$, $\Sigma$ is clopen.

Let $K$ be any compact subset of $X$, e.g., $K_{\alpha}$ for some $\alpha$. Then $K \cap \Sigma$ is compact, and there exists a positive integer $N$ such that $K \cap \Sigma=K \cap K_{\alpha_{N}}$ since $\Sigma=\bigcup_{n} K_{\alpha_{n}}^{0}$. Therefore $K \cap K_{\alpha_{n}}=K \cap K_{\alpha_{N}}$ for $n \geq N$.

We now choose sets $\left(L_{\beta}\right)_{\beta \in B}$ as in Lemma 2 and observe that this family of sets is a locally finite (called neighborhood-finite in [6]) cover of $X$ by pairwise disjoint, relatively compact sets. It follows easily that every open cover of $X$ has a (not 
necessarily open) locally finite refinement; thus $X$ is paracompact [6, Theorem 2.3 , p. 163].

REMARK. A slight modification of the preceding argument furnishes a new and simpler proof of Wheeler's WBAI theorem [13, Theorem 2.1]. Let $\left(h_{\alpha}\right)_{\alpha \in A}$ be a WBAI and, for each $\alpha$, let $K_{\alpha}$ be the (compact) support of the function $h_{\alpha}$. Let $<$ denote the given partial order on $A$. It is easy to see that Lemma 2 applies to the net $\left(K_{\alpha}\right)$. The preceding argument shows that $X$ is paracompact.

The next result does not invoke paracompactness of $X$, so it yields another proof of Theorem 3 via [13, Theorem 2.1].

Proposition 4. Let $X$ be a locally compact Hausdorff space. If $C_{0}(X)$ has a WCAI, then it has a WBAI.

Proof. Let $\left(f_{\alpha}\right)_{\alpha \in A}$ be a WCAI for $C_{0}(X)$ and, for each $\alpha$, let $K_{\alpha}=\left\{x: f_{\alpha}(x)\right.$ $\left.\geq \frac{1}{2}\right\}, B_{\alpha}=\left\{x: f_{\alpha}(x)>\frac{1}{3}\right\}$, and $C_{\alpha}=\left\{x: f_{\alpha}(x) \geq \frac{1}{3}\right\}$. Choose, for each $\alpha$, a continuous function $h_{\alpha}$ on $X$ such that $0 \leq h_{\alpha} \leq 1, h_{\alpha}=1$ on $K_{\alpha}$, and $h_{\alpha}=0$ on $X-B_{\alpha}$. Note that the (compact) support of $h_{\alpha}$ is a subset of $C_{\alpha}$. Put a (new) partial ordering < on $A$ as follows: $\alpha_{1}<\alpha_{2}$ if and only if $f_{\alpha_{2}}>\frac{3}{4}$ on $C_{\alpha_{1}}$.

To show that $\left(h_{\alpha}\right)_{\alpha \in A}$ is a WBAI, we need only check part (3) of the definition, since the rest of the definition clearly holds. Let $\alpha \in A, K$ be the support of $h_{\alpha}$, and $\left(\alpha_{n}\right)$ be a strictly increasing sequence in $A$. As in the proof of Theorem 3 , let $\Sigma=\bigcup_{n} K_{\alpha_{n}}=\bigcup_{n} K_{\alpha_{n}}^{0}$ and conclude that, for some positive integer $N, K \cap \Sigma=$ $K \cap K_{\alpha_{N}}$. Thus if $x \in K \cap \Sigma$, then $h_{\alpha_{n}}(x)=h_{\alpha_{N}}(x)=1$ for $n \geq N$. If $x \in K-\Sigma$, then $h_{\alpha_{n}}(x)=0$ for all $n$ since $\Sigma$ contains the support of $h_{\alpha_{n}}$ for all $n$. Thus $h_{\alpha} h_{\alpha_{n}}=h_{\alpha} h_{\alpha_{N}}$ for $n \geq N$ and, therefore, $\left(h_{\alpha}\right)_{\alpha \in A}$ is a WBAI.

We have seen that $X$ paracompact $\Rightarrow$ existence of a TBAI $\Rightarrow$ existence of a $\mathrm{WCAI} \Rightarrow$ existence of a WBAI $\Rightarrow X$ is paracompact. It is natural to inquire if every WBAI or WCAI contains a subnet which is a TBAI or, equivalently, which is equicontinuous. The concluding example shows that this need not be the case.

EXAMPLE 5. Let $N$ denote the set of positive integers, and let $X$ be the topological sum (union of disjoint spaces, each clopen in $X$ ) of the product space $N^{N}$, given the discrete topology, and sets $A_{1}, A_{2}, \ldots$, each of which is a copy of the closed unit interval $[0,1]$ with the usual topology. Observe that $X$ is locally compact Hausdorff and paracompact [6, Theorem 7.3, p. 241]. For each $n \in N$ and for each $x \in[0,1]$, let $h_{n}(x)=n x e^{-n x}$. Note that $h_{n}:[0,1] \rightarrow[0,1 / e]$ for each $n$ and that $h_{n} \rightarrow 0$ pointwise. Since $h_{n}(1 / n)=1 / e$, no subsequence of $\left(h_{n}\right)$ is equicontinuous at $x=0$.

Let us introduce some notation. Let $\pi_{n}: N^{N} \rightarrow N$ be the projection map onto the $n$th coordinate for each $n$. For each finite subset $S=\left\{a_{1}, \ldots, a_{n}\right\}$ of $N^{N}$, let $\varphi(S)=\sum \sum \pi_{i}\left(a_{j}\right)$ where the double summation is over the range $1 \leq i \leq n$, $1 \leq j \leq n$. Let $f_{S}: X \rightarrow[0,1]$ be defined as follows: $f_{S}=1$ on $S \cup A_{1} \cup \cdots \cup A_{n}$, $f_{S}=h_{\varphi(S)}$ on $A_{n+1}, f_{S}(x)=0$ for other $x$.

It is clear that each function $f_{S}$ is continuous and has compact support and that the net $\left(f_{S}\right)$, where the finite subsets $S$ are directed upward by inclusion, is an approximate identity for $C_{0}(X)$. Using a diagonal process, one can easily see that each sequence from the family $\left(f_{S}\right)$ has a subsequence which converges pointwise to an element of $C_{b}(X)$. Using Lemma 1 , we conclude that $\left(f_{S}\right)$ is a WCAI. It is also easily checked that $\left(f_{S}\right)$ is a WBAI. 
Now let $F \subset\left(f_{S}\right)$ be any equicontinuous subset. Because no infinite subset of the family $\left(h_{n}\right)$ is equicontinuous, for each $n \in N$ there exist $k_{n} \in N$ such that if $S=\left\{a_{1}, \ldots, a_{n}\right\} \subset N^{N}$ and $\varphi(S) \geq k_{n}$, then $f_{S} \notin F$. Let $x=\left(k_{1}, k_{2}, \ldots\right)$ and suppose that $f_{S}(x) \neq 0$ for some $S$ of cardinality $n$. Then $x \in S$ and $\varphi(S) \geq$ $k_{1}+\cdots+k_{n} \geq k_{n}$. Therefore $f_{S} \notin F$. Thus $f_{S}(x)=0$ for every $f_{S} \in F$ and, hence, $F$ is not an approximate identity for $C_{0}(X)$.

\section{REFERENCES}

1. R. C. Buck, Bounded continuous functions on a locally compact space, Michigan Math. J. 5 (1958), 95-104.

2. H. S. Collins, Strict, weighted, and mixed topologies and applications, Adv. in Math. 19 (1976), 207-237.

3. H. S. Collins and J. R. Dorroh, Remarks on certain function spaces, Math. Ann. 176 (1968), 157-168.

4. H. S. Collins and R. A. Fontenot, Approximate identities and the strict topology, Pacific J. Math. 43 (1972), 63-80.

5. J. B. Conway, The strict topology and compactness in the space of measures, Trans. Amer. Math. Soc. 126 (1967), 474-486.

6. J. Dugundji, Topology, Allyn and Bacon, Boston, Mass., 1966.

7. N. Dunford and J. T. Schwartz, Linear operators, Part I: General theory, Interscience, New York, 1958.

8. A. Grothendieck, Critères de compacité dans les espaces fonctionnels generaux, Amer. J. Math. 74 (1952), 168-186.

9 J. D. Pryce, $A$ device of $R$. J. Whitley's applied to pointwise compactness in spaces of continuous functions, Proc. London Math. Soc. (3) 23 (1971), 532-546.

10. I. Z. Ruzsa, personal communication, 1974.

11. F. D. Sentilles, Bounded continuous functions on a completely regular space, Trans. Amer. Math. Soc. 168 (1972), 311-336.

12. D. C. Taylor, A general Phillips theorem for $C^{*}$-algebras and some applications, Pacific J. Math. 40 (1972), 477-488.

13. R. F. Wheeler, Well-behaved and totally bounded approximate identities for $C_{0}(X)$, Pacific J. Math. 65 (1976), 261-269.

14. __ A survey of Baire measures and strict topologies, Exposition Math. 2 (1983), 97-190.

Department of MAThematics and Computer Science, Whitman College, WALLA WALLA, WASHINGTON 99362

DEPARTMENT OF MATHEMATICAL SCIENCES, NORTHERN ILLINOIS UNIVERSITY, DEKALB, ILLINOIS 60115 